\title{
Uncertainty Quantification of Crosstalk Using Stochastic Reduced Order Models
}

\author{
Zhouxiang Fei, Yi Huang, Senior Member, IEEE, Jiafeng Zhou, and Qian Xu
}

\begin{abstract}
This paper introduces a novel statistical method, referred to as the stochastic reduced order model (SROM) method, to predict the variability of cable crosstalk subject to a range of parametric uncertainties. The SROM method is a new member of the family of stochastic approaches to quantify propagated uncertainty in the presence of multiple uncertainty sources. It is non-intrusive, accurate, efficient, and stable, thus could be a promising alternative to some well-established methods such as the stochastic Galerkin (SG) and stochastic collocation (SC) methods. In this paper, the SROM method is successfully applied to obtain the statistics of cable crosstalk subject to single and multiple uncertainty sources. The statistics of uncertain cable parameters are first accurately approximated by SROMs, i.e., pairs of very few samples with known probabilities, such that the uncertain input space is well represented. Then, a deterministic solver is used to produce the samples of cable crosstalk with the corresponding probabilities, and finally the uncertainty propagated to the crosstalk is quantified with good accuracy. Compared to the conventional Monte Carlo (MC) simulation, the statistics of crosstalk obtained by the SROM method converge much faster by orders of magnitude. Also the computational cost of the SROM method is shown to be small and can be tuned flexibly depending on the accuracy requirement. The $\mathrm{SC}$ method based on tensor product sampling strategy is also implemented to validate the efficacy of the SROM method.
\end{abstract}

Index Terms - Cable crosstalk, electromagnetic compatibility, stochastic reduced order models, uncertainty quantification, variability analysis.

\section{INTRODUCTION}

$\mathrm{T}$ HE quality of the signal transmitted in cables is often degraded by the unintended interference from other wires in the cable or nearby cables. This interference, referred to as crosstalk, is caused by the interaction of electromagnetic fields generated by the currents along the wires or cables. The system may malfunction if the crosstalk exceeds the threshold. Therefore, the prediction of crosstalk is an important task to

Manuscript received November 11, 2015; accepted July 26, 2016. This work outlined above was carried out as part of the ICE-NITE project (see http://www.liv.ac.uk/icenite/), a collaborative research project supported by Innovate UK under contract reference 101665. (Corresponding author: Yi Huang.)

Z. Fei, Y. Huang, J. Zhou, and Q. Xu are with the Department of Electrical Engineering \& Electronics, The University of Liverpool, Liverpool, L69 3GJ, U.K. (e-mail: zhouxiang.fei@liv.ac.uk; yi.huang@liv.ac.uk). guarantee the cable performance from an early stage.

The fundamental approaches to deterministically calculate the crosstalk in the cables modeled by three-conductor transmission lines, multiconductor transmission lines (MTLs), and nonuniform MTLs (NMTLs) in the time and frequency domains were systematically addressed in [1]-[3]. For deterministic analysis, cable input variables were assumed to take deterministic values, i.e., these values truly represented input variables, and it only concerned how to obtain the exact crosstalk level. In such a case, the crosstalk level is unique.

However, the deterministic prediction of crosstalk is not enough, as uncertainties always exist in cable variables in reality [4], [32]. These uncertainties cause input variables to deviate from the nominal value. Therefore, the deterministic result obtained using the nominal input values may be unconvincing. This randomness feature of cables arises from many aspects, such as materials and cable routing. As a result, rather than having a specific crosstalk level, the output becomes a variation range consisting of all the possible crosstalk values caused by the uncertainties of input variables.

Due to the input uncertainties, statistical analyses were employed to predict the variation range and probability distribution of crosstalk [5]-[7], [32]. The traditional statistical approach is the brute Monte Carlo (MC) method [8]. For the MC method, a deterministic solver is needed to uniquely map input values to the corresponding output. Although being time-consuming, the MC method is non-intrusive as the existing deterministic solver is used without modifications. It is also general to all the uncertainty-embedded problems. Efforts have been made to simplify the statistical analysis. For example, the worst-case method was proposed in [9] to provide an envelope holding underneath all the possible variations of crosstalk. However, this method may be conservative as it overestimated crosstalk at non-resonant frequencies.

Recently, due to the breakthrough in uncertainty quantification methods, the polynomial chaos expansion (PCE) [10] and stochastic collocation (SC) methods [11], [16] have been intensively applied to obtain the statistics of crosstalk in the presence of input variability. The PCE method was able to describe crosstalk with an analytic formula regarding uncertain input variables [12]-[14]. The analytic formula was the sum of a series of orthogonal polynomials with proper coefficients obtained using the Stochastic Galerkin (SG) approach [25]. If the probability distributions of each uncertain variable were known, the statistics of crosstalk 
could be obtained by propagating input uncertainties to crosstalk using the probability theory in [15]. It is worth noting that in [12]-[14], the PCE-based SG method was intrusive as modifications to the existing deterministic solver were needed. However, the PCE method can also be implemented in a non-intrusive manner [26], [27]. It can be applied for uncertain variables with standard distributions, such as Gaussian distribution, or with arbitrary distributions [28]. Solving the stochastic model of the system could become a limitation for the PCE method when the number of uncertain variables increases, but this limitation can be significantly alleviated using decoupled and sparse PCE techniques [29], [31]. Also, PCE-based approaches can be directly applied to the transfer function of a system in electromagnetic simulators [27].

On the other hand, the SC method was non-intrusive and only required a selection of collocation points for each uncertain variable [19]. At collocation points, samples of crosstalk were obtained deterministically, and then interpolating functions were used to construct an analytical approximation of crosstalk, thus to recover statistics. However, the result of the SC method is sensitive to the choice of interpolating functions, as different interpolating functions produce slightly different output samples at the interpolated points adjacent to collocation points.

Very recently, the stochastic reduced order model (SROM) method was proposed in [18] as a potential alternative to the SG and SC methods to quantify propagated uncertainties in stochastic systems. The SROM method is conceptually simple, non-intrusive and efficient compared with the traditional MC method. The SROM method can be regarded as a small but smart version of the MC method, and therefore can be a general approach. It can be applied to uncertain variables with any types of distributions, and select input samples with regard to input distributions. An in-depth comparison between the SG, SC, and SROM methods was given in [19].

A SROM is an approximation of a random variable in the statistical sense, and has a small number of samples. Each sample is given a certain probability, such that the SROM and the random variable have similar statistics. To guarantee the performance, an objective function measuring the discrepancy between the statistics of the SROM and the random variable can be used. Once the SROM of uncertain input variables is constructed, the deterministic solver is used to obtain the SROM-based output response. Then, the statistics of the SROM-based output can be obtained with elementary calculations, and are used to approximate the statistics of the actual output. The SROM method has been used to solve uncertain mechanical engineering and material problems [20][23], but yet to be applied to electronic and electromagnetic compatibility (EMC) problems.

The aim of this paper is to present the first application of the SROM method to uncertainty-embedded EMC problems, in particular the uncertainty quantification of cable crosstalk. Given uncertain geometric variables of a cable, the statistics of crosstalk are obtained using the SROM method with a small computational cost, and the variation range is successfully bounded. The SC method implemented via tensor product sampling strategy is used as a reference to evaluate the performance of the SROM method. However, it is worth noting that more efficient SC implementations based on sparse grid sampling computed via the Smolyak algorithm are possible [31]. Therefore, the implemented SC method in this paper is not to represent the state-of-the-art SC method in terms of sampling requirements, and can only be used as a reference. The remainder of this paper is organized as follows: an overview of the SROM method is given in Section II. Section III describes the three-conductor transmission line as the cable model, and defines uncertain cable variables and crosstalk. Section IV presents the implementation of the SROM method to predict the statistics of crosstalk, and the result is compared to those of the SC and MC methods. Finally, the conclusion of the paper is given in Section V.

\section{Stochastic Reduced ORder Models (SROMS)}

In this section, the background of the SROM method is presented. First, the definition of a random variable is given. Let $\boldsymbol{X}$ be a $D$-dimensional random variable $(D \geq 1)$ if $\boldsymbol{X}$ is jointly described by $D$ variables. For example, if $\boldsymbol{X}$ is a bivariate random variable, i.e., $\boldsymbol{X}=\left[X_{1}, X_{2}\right]$, then $D=2$. It is assumed that the statistical properties of $\boldsymbol{X}$ are fully known beforehand, which are marginal distributions, moments of order $q$, and correlation matrix denoted as [23]:

$$
\begin{gathered}
F_{i}(\theta)=P\left(X_{i} \leq \theta\right) \\
\mu_{i}(q)=E\left(X_{i}^{q}\right) \\
\boldsymbol{r}=E\left[\boldsymbol{X} \boldsymbol{X}^{T}\right]
\end{gathered}
$$

where $i=1, \ldots, D$.

\section{A. Introduction to SROMs}

A SROM $\widetilde{\boldsymbol{X}}$ is an approximation of the random variable $\boldsymbol{X}$ in the sense that $\widetilde{\boldsymbol{X}}$ and $\boldsymbol{X}$ have similar statistical properties. The SROM $\widetilde{\boldsymbol{X}}$ consists of a sample set $\tilde{\boldsymbol{x}}=\left\{\tilde{\boldsymbol{x}}^{(1)}, \ldots, \tilde{\boldsymbol{x}}^{(m)}\right\}$ with the corresponding probabilities $\boldsymbol{p}=\left(p^{(1)}, \ldots, p^{(m)}\right)$ for each sample in $\tilde{\boldsymbol{x}}$. Any sample $\tilde{\boldsymbol{x}}^{(k)}, 1 \leq k \leq m$, contains one or multiple values depending on the dimension $D$ of $\boldsymbol{X}$, as $\tilde{\boldsymbol{x}}^{(k)}=$ $\left(\tilde{x}_{1}^{(k)}, \ldots, \tilde{x}_{D}^{(k)}\right)$. The elements in $\boldsymbol{p}$ are required to meet the constraints $\sum_{k=1}^{m} p^{(k)}=1$ and $p^{(k)} \geq 0$. Once the sample set $\tilde{\boldsymbol{x}}$ and probabilities $\boldsymbol{p}$ are selected, the SROM $\widetilde{\boldsymbol{X}}$ is completely defined. The model size $m$ is determined by the trade-off between accuracy and computational cost. A large value of $m$ usually gives very accurate statistical approximation of a random variable, whereas makes the implementation very computationally intensive [18]. Similar to $\boldsymbol{X}$, the statistics of the SROM $\widetilde{\boldsymbol{X}}$ are defined as:

$$
\begin{gathered}
\tilde{F}_{i}(\theta)=P\left(\tilde{X}_{i} \leq \theta\right)=\sum_{k=1}^{m} p^{(k)} \boldsymbol{I}\left(\tilde{x}_{i}^{(k)} \leq \theta\right) \\
\tilde{\mu}_{i}(q)=E\left(\tilde{X}_{i}^{q}\right)=\sum_{k=1}^{m} p^{(k)}\left(\tilde{x}_{i}^{(k)}\right)^{q} \\
\tilde{r}_{i j}=E\left[\tilde{X}_{i} \tilde{X}_{j}\right]=\sum_{k=1}^{m} p^{(k)} \tilde{x}_{i}^{(k)} \tilde{x}_{j}^{(k)}
\end{gathered}
$$


where $\boldsymbol{I}(A)=1$ if $A$ is true and $\boldsymbol{I}(A)=0$ if $A$ is false. Any sample set $\tilde{\boldsymbol{x}}$ and probabilities $\boldsymbol{p}$ can construct a SROM for $\boldsymbol{X}$. However, some SROMs produce more accurate approximation of the statistics of $\boldsymbol{X}$. For this reason, a way of measuring the discrepancy between $\widetilde{\boldsymbol{X}}$ and $\boldsymbol{X}$ in the statistical sense is needed. The next section describes how to construct an optimal SROM $\widetilde{\boldsymbol{X}}$ for $\boldsymbol{X}$ so that the discrepancy is minimized.

\section{B. Construction of SROMs}

There exist many SROMs for the random variable $\boldsymbol{X}$ as long as the sample-probability pair $\{\tilde{\boldsymbol{x}}, \boldsymbol{p}\}$ meets the constraints in the previous section. However, to implement the SROM method, an optimal SROM $\widetilde{\boldsymbol{X}}$ for the input variable $\boldsymbol{X}$ is required so that the discrepancy between the statistics of $\widetilde{\boldsymbol{X}}$ and $\boldsymbol{X}$ is minimized. The discrepancy is measured with an objective function containing three error metrics. These error metrics represent the errors between marginal distributions, moments up to order of $\bar{q}$, and correlation matrices of $\widetilde{\boldsymbol{X}}$ and $\boldsymbol{X}$, respectively, and are defined as:

$$
\begin{gathered}
e_{1}(\widetilde{\boldsymbol{x}}, \boldsymbol{p})=\sum_{i=1}^{D} \sum_{k=1}^{m}\left(\tilde{F}_{i}\left(\tilde{x}_{i}^{(k)}\right)-F_{i}\left(\tilde{x}_{i}^{(k)}\right)\right)^{2} \\
e_{2}(\widetilde{\boldsymbol{x}}, \boldsymbol{p})=\sum_{i=1}^{D} \sum_{q=1}^{\bar{q}}\left(\tilde{\mu}_{i}(q)-\mu_{i}(q)\right)^{2} \\
e_{3}(\widetilde{\boldsymbol{x}}, \boldsymbol{p})=\sum_{i, j=1, \ldots, D ; j>i}\left(\tilde{r}_{i j}-r_{i j}\right)^{2} .
\end{gathered}
$$

With each error metric defined, the objective function measuring the total discrepancy of statistics between $\widetilde{\boldsymbol{X}}$ and $\boldsymbol{X}$ can be expressed as:

$$
e(\widetilde{\boldsymbol{x}}, \boldsymbol{p})=\alpha_{1} e_{1}(\widetilde{\boldsymbol{x}}, \boldsymbol{p})+\alpha_{2} e_{2}(\widetilde{\boldsymbol{x}}, \boldsymbol{p})+\alpha_{3} e_{3}(\widetilde{\boldsymbol{x}}, \boldsymbol{p})
$$

where $\alpha_{1}, \alpha_{2}, \alpha_{3} \geq 0$ are weighting factors to make each error metric having a similar order of magnitude, or to emphasize which statistical property of $\boldsymbol{X}$ to be approximated more accurately. For example, we can set $\alpha_{1} \gg \alpha_{2}$ and $\alpha_{3}$ if the marginal distribution of $\boldsymbol{X}$ needs to be approximated more precisely by $\widetilde{\boldsymbol{X}}$. The optimal SROM $\widetilde{\boldsymbol{X}}$ is defined by the sample-probability pair $\left\{\tilde{\boldsymbol{x}}_{\text {(opt) }}, \boldsymbol{p}_{(\mathrm{opt})}\right\}$ that minimizes the objective function in (10). As a result, this $\widetilde{\boldsymbol{X}}$ is the closest to $\boldsymbol{X}$ in the statistical sense. If $\boldsymbol{X}$ is a one-dimensional random variable, i.e., $D=1$, the error of correlation matrices in (9) can be ignored when constructing the optimal SROM $\widetilde{\boldsymbol{X}}$.

The pattern classification [18] is a common method to find the optimal SROM $\widetilde{\boldsymbol{X}}$, and is outlined in seven steps as follows:

Step 1): Generate a collection consisting of $n$ independent samples $\left(\boldsymbol{\xi}_{1}, \ldots, \boldsymbol{\xi}_{n}\right)$ for the random variable $\boldsymbol{X}$. The cardinality $n$ should be large enough to describe the statistics of $\boldsymbol{X}$ accurately.

Step 2): Randomly extract a subset $\left(\tilde{\boldsymbol{x}}^{(1)}, \ldots, \tilde{\boldsymbol{x}}^{(m)}\right)$ from $\left(\boldsymbol{\xi}_{1}\right.$, $\left.\ldots, \boldsymbol{\xi}_{n}\right), m \ll n$.

Step 3): Divide the uncertain region of $\boldsymbol{X}$ into $m$ Voronoi regions with $\left(\tilde{\boldsymbol{x}}^{(1)}, \ldots, \tilde{\boldsymbol{x}}^{(m)}\right)$ as generator seeds [30]. The Voronoi region $\Gamma_{k}$ centered at $\tilde{\boldsymbol{x}}^{(k)}(1 \leq k \leq m)$ is comprised of all the samples from $\left(\boldsymbol{\xi}_{1}, \ldots, \boldsymbol{\xi}_{n}\right)$ that are closest to $\tilde{\boldsymbol{x}}^{(k)}$ than to any other center $\tilde{\boldsymbol{x}}^{(l)}(l \neq k, 1 \leq l \leq m)$. Then, the Euclidean distance from $\xi_{i}(1 \leq i \leq n)$ to $\tilde{\boldsymbol{x}}^{(k)}$ is measured.

Step 4): Let $d^{(k)}$ represent the summation of the distances from all the samples in $\Gamma_{k}$ to the center $\tilde{\boldsymbol{x}}^{(k)}$, i.e., $d^{(k)}=$ $\sum_{i \in \Gamma_{k}} d\left(\widetilde{\boldsymbol{x}}^{k}, \xi_{i}\right)$. Then, calculate $d=\sum_{k=1}^{m} d^{(k)}$ as the overall distance between the subset $\left(\tilde{\boldsymbol{x}}^{(1)}, \ldots, \tilde{\boldsymbol{x}}^{(m)}\right)$ and $\left(\boldsymbol{\xi}_{1}, \ldots, \boldsymbol{\xi}_{n}\right)$.

Step 5): Select other candidate subsets $\left(\tilde{\boldsymbol{x}}^{(1)}, \ldots, \tilde{\boldsymbol{x}}^{(m)}\right)$ and calculate the values of $d$ for each subset.

Step 6): The sample set $\tilde{\boldsymbol{x}}_{\text {(opt) }}$ is selected as the subset $\left(\tilde{\boldsymbol{x}}^{(1)}\right.$, $\ldots, \tilde{\boldsymbol{x}}^{(m)}$ ) with the minimum value of $d$. As a result, the samples in $\tilde{\boldsymbol{x}}_{\text {(opt) }}$ are most widely separated to explore the entire uncertain region of $\boldsymbol{X}$.

Once $\tilde{\boldsymbol{x}}_{(\mathrm{opt})}$ is determined, the probability set $\boldsymbol{p}_{(\mathrm{opt})}$ can be obtained in one step as shown below:

Step 7): Let $n_{k}$ denote the number of the samples in $\Gamma_{k}, 1 \leq k$ $\leq m$. The probability for $\tilde{\boldsymbol{x}}^{(k)}$ is calculated as $p^{(k)}=n_{k} / n$. Thus, the probability set $\boldsymbol{p}_{\text {(opt) }}$ can be obtained as the set $\left\{p^{(k)}\right\}_{k=1}^{m}$.

With $\tilde{\boldsymbol{x}}_{\text {(opt) }}$ and $\boldsymbol{p}_{\text {(opt) }}$ obtained, the optimal SROM $\widetilde{\boldsymbol{X}}$ is defined as the sample-probability pair $\left\{\tilde{\boldsymbol{x}}_{(\mathrm{opt})}, \boldsymbol{p}_{(\mathrm{opt})}\right\}$.

\section{Uncertainty Propagation by SROMs}

A workflow illustrating how the uncertainty is propagated from the random input variable $\boldsymbol{X}$ to the output $\boldsymbol{Y}$ with the SROM method is outlined in Fig. 1. The statistics of the actual output $\boldsymbol{Y}$ are approximated by those of the SROM-based output $\widetilde{\boldsymbol{Y}}$. The construction of $\widetilde{\boldsymbol{Y}}$ requires an optimal SROM $\widetilde{\boldsymbol{X}}$ $=\{\tilde{\boldsymbol{x}}, \boldsymbol{p}\}$ for $\boldsymbol{X}$ and a deterministic solver $M$. The deterministic solver is used to produce the samples of the output $\boldsymbol{Y}$ given the samples of the input $\boldsymbol{X}$. Similar to $\widetilde{\boldsymbol{X}}, \widetilde{\boldsymbol{Y}}$ is also defined by a sample set $\tilde{\boldsymbol{y}}=\left\{\tilde{\boldsymbol{y}}^{(1)}, \ldots, \tilde{\boldsymbol{y}}^{(m)}\right\}$ together with the corresponding probabilities $\boldsymbol{p}_{y}=\left(p_{y}{ }^{(1)}, \ldots, p_{y}{ }^{(m)}\right)$. With the samples $\left\{\widetilde{\boldsymbol{x}}^{(i)}\right\}_{i=1}^{m}$ for $\widetilde{\boldsymbol{X}}$ known, the samples $\left\{\widetilde{\boldsymbol{y}}^{(i)}\right\}_{i=1}^{m}$ for $\widetilde{\boldsymbol{Y}}$ can be obtained by performing $m$ deterministic calculations with the variable $\boldsymbol{X}$ set equal to $\tilde{\boldsymbol{x}}^{(1)}, \ldots, \tilde{\boldsymbol{x}}^{(m)}$ :

\section{Uncertain input variable $\boldsymbol{X}$}

Described by: $\xi_{1}, \xi_{2}, \ldots, \xi_{n}$. Cardinality: $n$

$$
\downarrow \text { Approximated by } \widetilde{\boldsymbol{X}}
$$

\begin{tabular}{|c|c|}
\hline Samples: $\widetilde{\boldsymbol{y}}$ & $\widetilde{\boldsymbol{y}}^{(1)}, \widetilde{\boldsymbol{y}}^{(2)}, \ldots, \widetilde{\boldsymbol{y}}^{(m)}$ \\
\hline Probabilities: $p_{y}$ & $p_{y}{ }^{(1)}, p_{y}^{(2)}, \ldots, p_{y}^{(m)}$ \\
\hline
\end{tabular}

Optimal SROM $\widetilde{\boldsymbol{X}}$, Cardinality: $m, m \ll n$

\begin{tabular}{|c|l|}
\hline Samples: $\widetilde{\boldsymbol{x}}$ & $\widetilde{\boldsymbol{x}}^{(1)}, \widetilde{\boldsymbol{x}}^{(2)}, \ldots, \widetilde{\boldsymbol{x}}^{(m)}$ \\
\hline Probabilities: $\boldsymbol{p}$ & $p^{(1)}, p^{(2)}, \ldots, p^{(m)}$ \\
\hline
\end{tabular}

$$
\downarrow \text { Via the deterministic solver } M
$$

SROM-based solution/output $\widetilde{\boldsymbol{Y}}$

Fig. 1. Workflow of propagating uncertainty from the input variable $X$ to the output $\boldsymbol{Y}$ with the SROM method. 


$$
M: \widetilde{\boldsymbol{x}}^{(k)} \rightarrow \widetilde{\boldsymbol{y}}^{(k)}, k=1, \ldots, m .
$$

The probabilities $\boldsymbol{p}_{y}$ of $\widetilde{\boldsymbol{Y}}$ are the same as the probabilities $\boldsymbol{p}$ of $\widetilde{\boldsymbol{X}}$, i.e., $p_{y}{ }^{(k)}=p^{(k)}, k=1, \ldots, m$. The reason is that $\tilde{\boldsymbol{y}}^{(k)}$ only occurs when the input is $\tilde{\boldsymbol{x}}^{(k)}$. Having obtained the sample set $\tilde{\boldsymbol{y}}$ and probabilities $\boldsymbol{p}_{y}$, the SROM-based solution $\widetilde{\boldsymbol{Y}}$ is completely defined. The calculation of the statistics of $\widetilde{\boldsymbol{Y}}$, such as distributions and moments of order $q$, becomes an easy task as shown below:

$$
\begin{gathered}
P(\tilde{Y} \leq \xi)=\sum_{k=1}^{m} p^{(k)} \boldsymbol{I}\left(\tilde{y}^{(k)} \leq \xi\right) \\
E\left(\tilde{Y}^{q}\right)=\sum_{k=1}^{m} p^{(k)}\left(\tilde{y}^{(k)}\right)^{q} .
\end{gathered}
$$

The standard deviation $\sigma$ for $\widetilde{\boldsymbol{Y}}$ can be obtained using:

$$
\sigma(\tilde{Y})=\sum_{k=1}^{m} p^{(k)}\left(\tilde{y}^{(k)}-E\left(\tilde{Y}^{1}\right)\right)^{2} .
$$

The statistics of $\boldsymbol{Y}$ are approximated by those of $\widetilde{\boldsymbol{Y}}$ in (12)(14). The SROM method can be an a-priori evaluation by developing the error bound of the SROM solution for different model sizes as in [20], [21], which is beyond the scope of this paper. Increasing the model size $m$ is an effective way to reduce the error of the SROM result, but choosing the value of $m$ mainly depends on the consideration of computation time. In principle, the SROM solution is guaranteed to converge to the theoretical statistics of $\boldsymbol{Y}$ when the model size $m$ approaches infinity [17]. Despite this, the SROM method has been shown to be able to produce very accurate statistics, even with a small $m$ to reduce the computational cost [17], [23]. It is clear that the SROM method has the non-intrusive feature and is therefore very convenient to implement. This method is also very efficient compared to the traditional MC simulation, as the effect of the uncertain input space on the output variation is taken into account using only $m$ samples and the corresponding probabilities. The only overhead is to construct the optimal $\widetilde{\boldsymbol{X}}$ to ensure that the statistics of the input variable $\boldsymbol{X}$ are accurately approximated.

In summary, to propagate the uncertainty from the input variable $\boldsymbol{X}$ to the output $\boldsymbol{Y}$ using the SROM method, only three steps are needed. First, an optimal SROM $\widetilde{\boldsymbol{X}}$ for $\boldsymbol{X}$ is constructed to minimize (10). This step is the nucleus of the SROM method, and totally isolated from the deterministic solver. Second, the SROM-based output $\widetilde{\boldsymbol{Y}}$ for $\boldsymbol{Y}$ is constructed using $\widetilde{\boldsymbol{X}}$ and the deterministic solver. Finally, the statistics of $\widetilde{\boldsymbol{Y}}$ are calculated to approximate those of the actual output $\boldsymbol{Y}$.

\section{CABLE MODEL}

In this section, the input variables, output responses, and deterministic solver of the cable model are introduced, as these three aspects are involved in the SROM method. In this study, the cable bundle is modeled as a three-conductor transmission line. Due to the well-established deterministic solver of this model, it has been used to validate the efficacy of the SG and SC methods for predicting the statistics of crosstalk in [12], [16]. Therefore, the three-conductor transmission line is also chosen to verify the efficacy of the

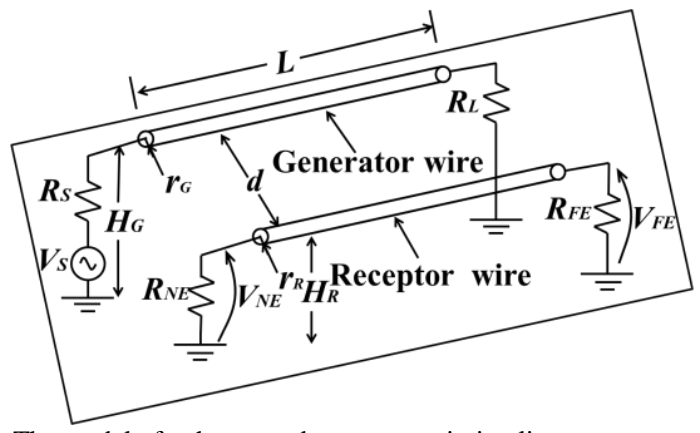

Fig. 2. The model of a three-conductor transmission line.

SROM method for quantifying the uncertainty propagated from input variables to crosstalk.

\section{A. Input Variables}

Fig. 2 shows the schematic of a three-conductor transmission line. The two parallel conductors with length $L$ are known as the generator wire and the receptor wire. As the names indicate, the generator wire could induce crosstalk on the receptor wire. The third conductor is the ground to which voltages and the heights of wires are referenced. In the generator circuit, the generator wire connects a voltage source $V_{S}$ with impedance $R_{S}$ to a load with impedance $R_{L}$. In the receptor circuit, the termination loads $R_{N E}$ and $R_{F E}$ at two ends are connected by the receptor wire. The subscripts $N E$ and $F E$ indicate if the load is at the near-end or far-end of the receptor circuit. The return paths of the generator and receptor circuits are formed by the ground.

Apart from the electrical parameters mentioned above, the crosstalk is also determined by the following geometric variables: the wire length $L$, the radius $r_{G}$ and height $H_{G}$ of the generator wire, the radius $r_{R}$ and height $H_{R}$ of the receptor wire, and the distance $d$ between the generator and receptor wires. The following assumptions are used: $r_{G}=r_{R}=r, H_{G}=$ $H_{R}=H$, and $R_{S}=R_{L}=R_{N E}=R_{F E}=T$.

\section{B. Output Responses}

When switching on the source $V_{S}$, the coupled voltages $V_{N E}$ and $V_{F E}$ are induced to the near-end load $R_{N E}$ and far-end load $R_{F E}$ in the receptor circuit, respectively. The crosstalk is defined as the ratio of the induced voltage to the source voltage [5]:

$$
N E X T=\frac{V_{N E}}{V_{S}}, F E X T=\frac{V_{F E}}{V_{S}}
$$

where NEXT means the near-end crosstalk and FEXT means the far-end crosstalk. The output responses of the cable model are NEXT and FEXT.

\section{Deterministic Solver}

The traditional deterministic solver for calculating crosstalk is the Telegrapher's equations used in [12]. An analytical solution was derived in [24] to directly calculate crosstalk based on the values of input variables, thus to bypass solving the Telegrapher's equations. Therefore, this analytical solution is used as the deterministic solver with the following assumptions: 1) the two wires and ground are made of perfect electric conductors; 2) the cross-sections of two wires are 
TABLE I

DETERMINISTIC VALUES OF INPUT VARIABLES

\begin{tabular}{cc}
\hline \hline Input variable & Deterministic value \\
\hline$L(\mathrm{~m})$ & 7 \\
$r(\mathrm{~mm})$ & 1.024 \\
$d(\mathrm{~mm})$ & 6 \\
$T(\Omega)$ & 50 \\
$f(\mathrm{MHz})$ & 400 \\
$H(\mathrm{~mm})$ & 10 \\
\hline
\end{tabular}

invariant along the cable length; 3) the medium around wires is lossless and homogeneous.

\section{APPLICATIONS OF SROMS}

In this section, the SROM method is applied to obtain the statistics of crosstalk in the presence of single or multiple uncertain variables. To propagate uncertainty with the SROM method, the first step is to construct a SROM for uncertain cable variables. Then, the SROM-based output $\widetilde{N E X T}$ and $\widetilde{F E X T}$ for the actual output NEXT and FEXT can be constructed with the deterministic solver. Finally, the statistics of NEXT and FEXT are approximated by those of $\widetilde{N E X T}$ and $\widetilde{F E X T}$ using (12)-(14). In subsequent sections, the NEXT is considered as the output in one example, and then the FEXT is used in the other example. For the demonstration purpose, uncertain cable variables are assumed to have Gaussian distributions. However, it is worth noting that the SROM method is applicable for any types of probability distributions, and switching from one type of probability distributions to another is straightforward. The SROM method is demonstrated with three examples where the number of random variables gradually increases. The frequency at which the simulation was run is set to $400 \mathrm{MHz}$. To validate the SROM method, the SROM-based result is compared to those of the MC method and the SC implementation based on tensor product sampling strategy. The statistics from 1,000,000 MC simulations are used as reference results to set benchmarks.

\section{A. Single Uncertainty Source: Height H}

The aim of this section is to demonstrate the implementation of the SROM method for a single uncertainty variable: the wire height $H$ with a Gaussian distribution of mean $E(H)=10 \mathrm{~mm}$ and standard deviation $\sigma(H)=1 \mathrm{~mm}$. Other variables are regarded to take deterministic values shown in Table I.

The construction of the optimal $\widetilde{H}$ for $H$ follows the guideline described in Section II (B). As $H$ is a 1-dimensional random variable, there is no need to consider the discrepancy in correlation matrices in (9) when constructing $\widetilde{H}$. Three optimal SROMs $\widetilde{H}$ are constructed with 5, 10, and 20 samples, respectively, and are used to approximate the cumulative distribution function (CDF) of $H$ denoted by $\mathrm{F}(H)$ in Fig. 3(a). It is clear that as the sample size of $\widetilde{H}$ increases, the approximated probability distribution of $H$ becomes closer to the reference distribution. When the sample size is 20 , the difference between the reference and SROM-based
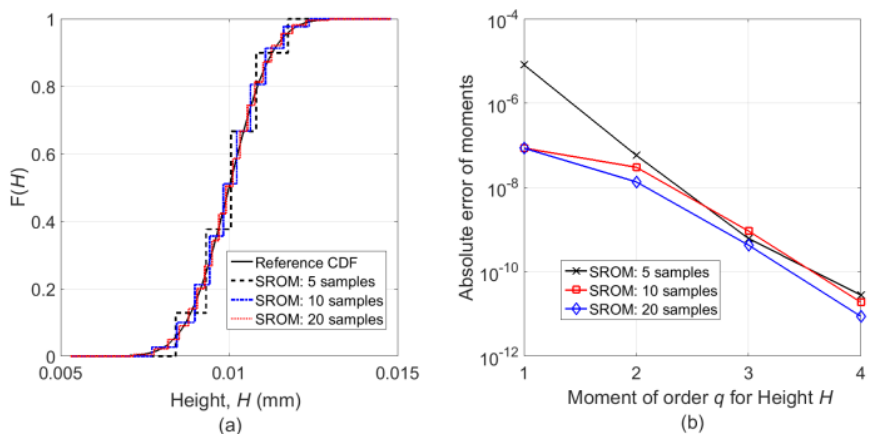

Fig. 3. (a) The reference CDF of the uncertain variable $H$ and the CDFs approximated by the SROMs $\widetilde{H}$ formed with 5,10 , and 20 samples. (b) Absolute errors of moments approximated by SROMs $\widetilde{H}$ with sizes of 5, 10, and 20 .

distributions is very small.

Fig. 3(b) shows the absolute error of moments up to the order of 4 for $\widetilde{H}$ constructed with 5, 10, and 20 samples. Generally speaking, the error at each moment order is reduced by increasing the sample size of $\widetilde{H}$. It is seen that the model size of 10 can provide an accurate approximation for each moment order, and increasing the size from 10 to 20 does not further reduce the error significantly. This nice feature means that the SROM method does not need a very large sample size to achieve good accuracy. In the case of uncertain $H$, a sample size of 10 is reasonable as the approximated CDF and moment orders match the reference counterparts in good agreement, and the computational cost is kept low.

After the SROMs $\widetilde{H}$ with sizes of 5, 10, and 20 samples are constructed, the deterministic solver is used to produce the samples of the SROM-based output $\widetilde{N E X T}$. Due to the one-toone relationship between the input and output samples, the sample sizes of the three corresponding $\widehat{N E X T}$ are also 5, 10, and 20, respectively. The probabilities of the samples in $\widetilde{N E X T}$ are the same as those in $\widetilde{H}$. With the samples and probabilities obtained, the SROM-based solution $\widehat{N E X T}$ can be constructed. In Fig. 4, the CDFs of $\widetilde{N E X T}$ are plotted to
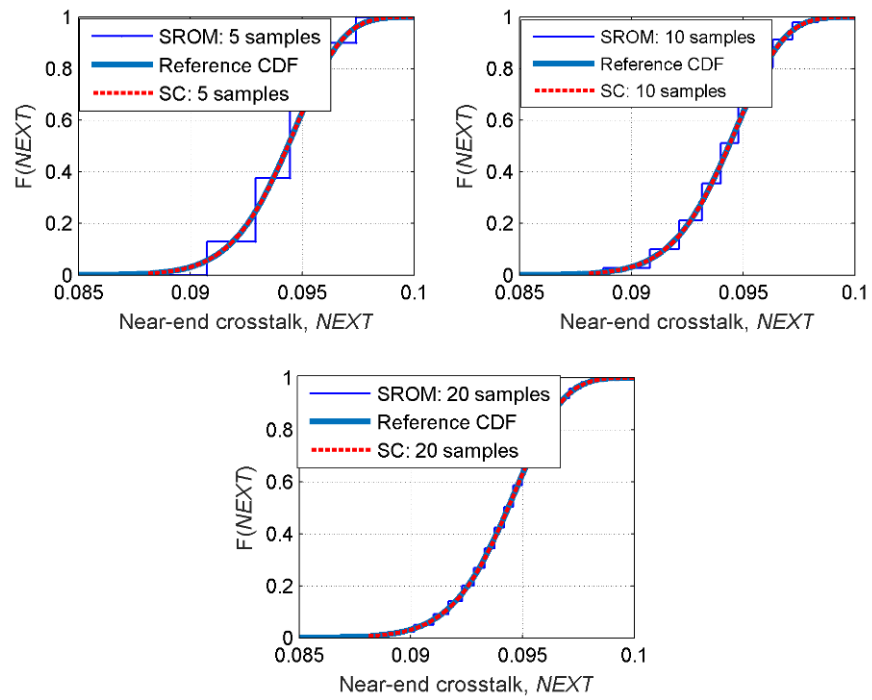

Fig. 4. The reference CDF of NEXT, the CDFs approximated by the SC method with 5, 10, and 20 collocation points, and the CDFs approximated by the SROMs $\widetilde{N E X T}$ with sample sizes of 5, 10, and 20. 

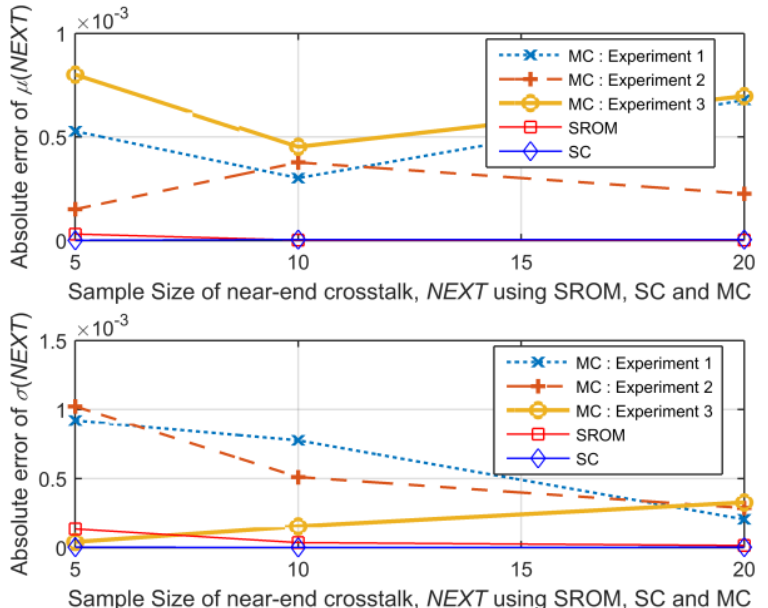

Fig. 5. Absolute errors of the statistics of NEXT obtained by the SROM, SC, and MC methods using small sample sizes.

approximate the reference CDF of the actual NEXT. It is seen that all three $\widetilde{N E X T}$ are able to recover the general shape of the reference distribution, and the $\widetilde{N E X T}$ with 20 samples gives the closest distribution for NEXT. This is because the corresponding input $\widetilde{H}$ with size of 20 provides the most accurate statistics for $H$. Therefore, the performance of the SROM method is highly dependent on the quality of the input SROM. On the other hand, the SC method using Lagrange polynomials as the interpolating function is also implemented to compare with the SROM method. For the SC method of this study, the number of collocation points is chosen the same as the sample size of the SROM method, such that the deterministic solver is run with the same number of times by the two methods. As can be seen in Fig. 4, unlike the stepshaped CDFs given by the SROM method, the SC method can produce faultless and continuous CDFs for NEXT using 5, 10, and 20 collocation points.

In addition to providing the distribution information, the SROM method is also able to predict the mean and standard deviation of NEXT with great accuracy. As Fig. 5 shows, the mean value $\mu$ (NEXT) and standard deviation $\sigma$ (NEXT) given by $\overparen{N E X T}$ with different sizes are very accurate, and the accuracy is improved by increasing the sample size of $\widetilde{N E X T}$, but not dramatically. This is because in this case the approximated statistics by the SROM method converge to the reference values very fast. At the sample size of 10, the mean value and standard deviation given by the SROM method are almost identical to the reference counterparts. In contrast to the SROM method, three MC experiments are performed, and each MC experiment is performed with 5, 10, and 20 samples. As shown in Fig. 5, from size of 5 to 20, the variation of the statistics given by the MC method is different from one experiment to another. At the size of 20 , the mean and standard deviation by the MC method fail to converge to the reference results as close as the SROM method. We note that in the MC experiment 3 with the size of 5 , an accurate standard deviation could be produced by incident, but the accuracy is unrepeatable. Therefore, at small sample sizes, the MC method is inaccurate and gives different results when

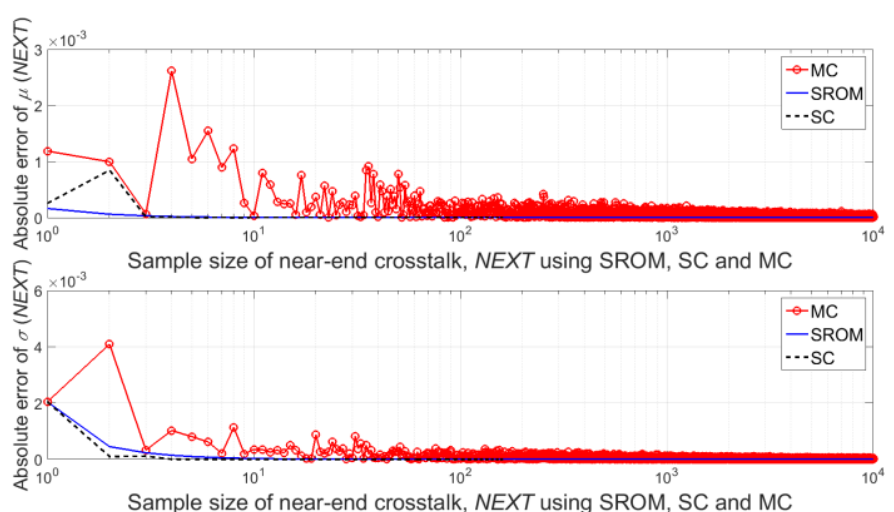

Fig. 6. Convergence rates of the SROM, SC and MC methods to the reference statistics of NEXT.

repeating experiment. It is worth noting that the corresponding confidence interval for each moment can be estimated based on the sample size of the MC simulation, which is beyond the scope of this study. By contrast, the SROM method is stable as long as the uncertain input space is well approximated by SROMs, and able to provide very accurate mean using small sample sizes. On the other hand, the SC method can produce almost error-free statistics using only 5 collocation points, but the difference between the accuracies of the SROM and SC methods is very small in this example.

Fig. 6 demonstrates the convergence rates of the SROM, SC and $\mathrm{MC}$ methods to produce accurate statistics of NEXT. As can be seen, both the SROM and SC methods converge to the reference result faster than the MC method. Specifically, the MC method needs at least $10^{4}$ samples to converge to the accuracy of the SROM method at sample size of 10 . Therefore, comparing with MC, the SROM method reduces the computational cost by a factor of $10^{4} / 10=10^{3}$ in this case, which is a sizable acceleration for stochastic analysis. On the other hand, only 4 collocation points are needed by the SC method to give the same performance of the SROM method with size of 10. However, the relative goodness between the SC and SROM methods cannot be purely evaluated using the sample size needed for certain accuracy. This is because for the SC method, after obtaining the output samples at collocation points, the overhead is to derive the analytical approximation of the output response using the interpolating function, and then the statistics of the output can be recovered. By contrast, for the SROM method, after the SROM-based output is obtained, only elementary calculation in (12) - (14) is needed to recover the statistics of the output. It is clear that in the presence of single uncertain source, both the SROM and SC methods are efficient to produce the accurate statistics of crosstalk, as only a small fraction of the computational cost of the $\mathrm{MC}$ method is required.

In Fig. 7, the reference probability distribution function (PDF) of NEXT is plotted to compare with the probabilities of the samples in $\widetilde{N E X T}$ with sample size of 10 . It is clear that the discrete probabilities of $\widetilde{N E X T}$ are in good agreement with the shape of the reference PDF. Therefore, the probability of each sample in $\widetilde{N E X T}$ can reflect the possibility of the actual $N E X T$ taking values in the vicinity of this sample. On the other 


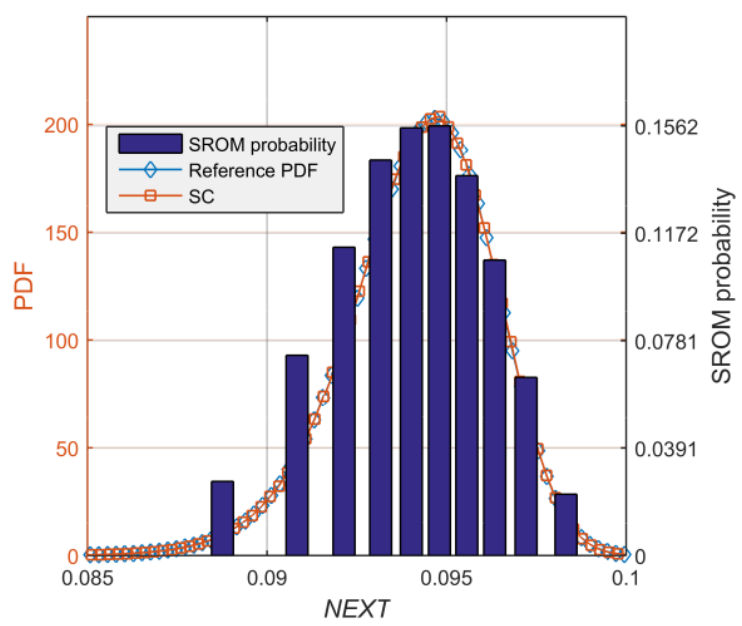

Fig. 7. The reference PDF of the output NEXT, the PDF obtained by the SC method with 10 collocation points, and the probabilities of the samples in the SROM-based $\widehat{N E X T}$ with sample size of 10 .

hand, the PDF approximated by the SC method with 10 collocation points is exactly the same as the reference PDF. Therefore, the SC method may be a better approach if the aim is to recover the output PDF in detail.

As the SROM method can predict the accurate mean $\mu$ and standard deviation $\sigma$ of NEXT, the variation range of NEXT can be bounded as the interval: $[\mu-3 \sigma, \mu+3 \sigma]$. The boundaries of the NEXT variations are obtained by the SROM method and plotted from $1 \mathrm{MHz}$ to $400 \mathrm{MHz}$ in Fig. 8. It can be seen that only a small number of extreme cases are outside the variation range. It is worth noting that only 10 samples of $\widetilde{N E X T}$ are required at each frequency to obtain the variation range. Therefore, the SROM method is able to predict the accurate variation range of crosstalk with small computational cost.

\section{B. Two Uncertainty Sources}

In this section, the SROM method is applied in the presence of two random variables: the wire height $H$ and distance $d$

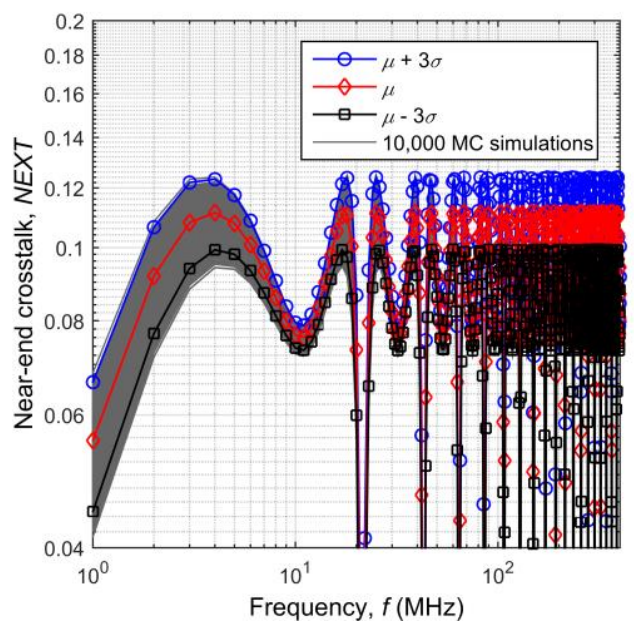

Fig. 8. Upper and lower boundaries obtained using the SROM method to bound the variation of NEXT. At each frequency, only 10 samples of the SROM-based $\widetilde{N E X T}$ are used. The uncertain variable is $H$.

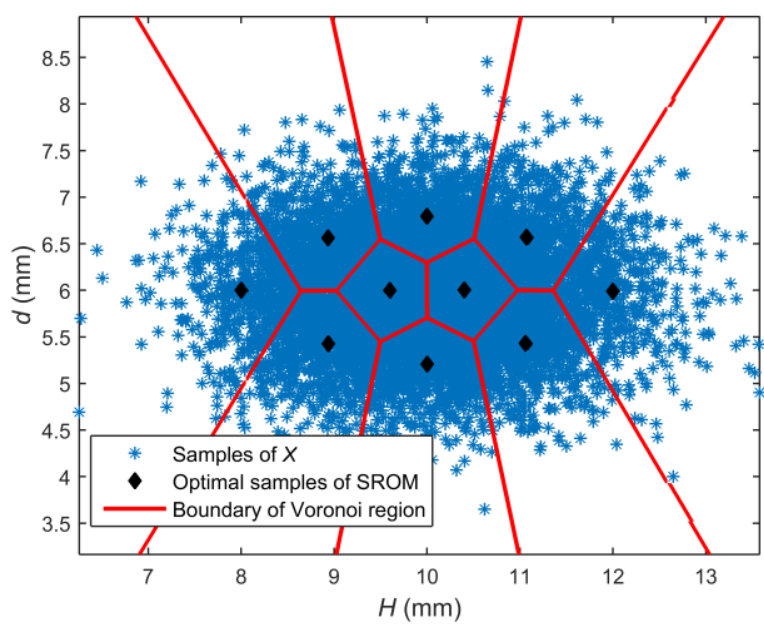

Fig. 9. The distribution of 10,000 samples of $\boldsymbol{X}$, and 10 optimal samples of $\widetilde{\boldsymbol{X}}$ in corresponding Voronoi regions.

between two wires. To tackle multiple uncertainty sources with the SROM method, the idea is to regard each uncertainty source as a 1-dimensional variable, and integrate these uncertainty sources into a multidimensional variable. Then, a SROM can be constructed for this multidimensional variable to globally approximate the overall uncertain input space. For example, we can use the $D$-dimensional random variable $\boldsymbol{X}$ described in Section II to contain two 1-dimensional variables $H$ and $d$, i.e., $\boldsymbol{X}=[H, d]$. In this case, $\boldsymbol{X}$ is a bivariate variable and $D=2$. Each sample of $\boldsymbol{X}$ represents a point in a plane formed with $H$ as the $x$-axis and $d$ as the $y$-axis. The coordinates of the point contains a set of possible values of $H$ and $d$ to run the deterministic solver once. As a result, the uncertainties of $H$ and $d$ can be jointly approximated by building a SROM for $\boldsymbol{X}=[H, d]$.

The height $H$ and distance $d$ are assumed to follow the Gaussian distribution with the mean values $E(H)=10 \mathrm{~mm}$ and $E(d)=6 \mathrm{~mm}$, and the standard deviations $\sigma(H)=1 \mathrm{~mm}$ and $\sigma(d)=0.6 \mathrm{~mm}$. Other variables are considered as deterministic values in Table I. A SROM $\widetilde{\boldsymbol{X}}$ with a sample size of 10 is used to visualize the concept of the SROM of 2-dimensional variable $\boldsymbol{X}=[H, d]$. Fig. 9 shows the distribution of 10,000 samples of $\boldsymbol{X}$. In addition, 10 optimal samples of $\widetilde{\boldsymbol{X}}$ are selected from the 10,000 samples of $\boldsymbol{X}$ using the algorithm introduced in Section II(B), and are plotted in the Voronoi tessellation in Fig. 9. As these 10 samples of $\widetilde{\boldsymbol{X}}$ are widely separated from each other, the entire uncertain region of $\boldsymbol{X}$ is explored, rather than only focusing on highly likely or marginal regions.

The probability of each optimal sample in $\widetilde{\boldsymbol{X}}$ can be calculated using the number of samples in the corresponding Voronoi region. Having obtained the sample and probability sets, the optimal SROM $\widetilde{\boldsymbol{X}}$ is constructed and visualized versus the PDF of $\boldsymbol{X}$ in Fig. 10. As shown in Fig. 10, the coordinates of a red dot on the $H-d$ plane indicate the values of $H$ and $d$ contained in this optimal sample, and the height of the red dot represents the corresponding probability. 


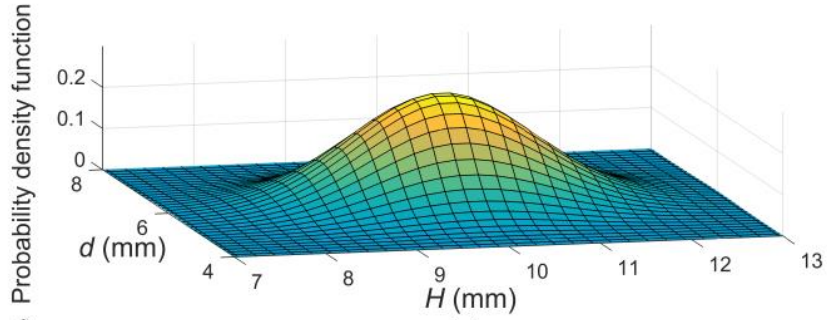

(a)

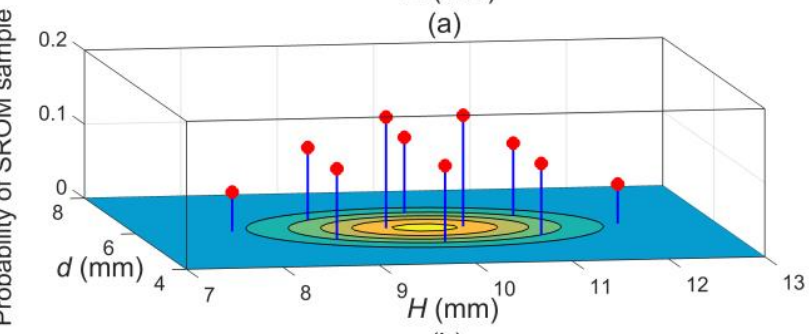

(b)

Fig. 10. (a) The PDF of a bivariate variable $X=[H, d]$. (b) The visualization of an optimal SROM $\widetilde{\boldsymbol{X}}$ with sample size of 10 .

Both the SROM and SC methods are used to propagate the uncertainty from $X=[H, d]$ to $N E X T$. In this example, the SC method based on tensor product sampling is implemented using the cubic Hermite interpolating function [33]. The number of collocation points on the $H-d$ plane is set to $3 \times 3,4$ $\times 4$ and $5 \times 5$, respectively. Here, $3 \times 3$ means there are 3 collocation points in the uncertain ranges of $H$ and $d$, respectively. To ensure the deterministic solver is evaluated by the SROM method with the same number of times, the sample size of the SROM $\widetilde{\boldsymbol{X}}$ is set to 9, 16 and 25 accordingly. At each sample size, the predicted CDFs of NEXT using the SROM and SC methods are plotted in Fig. 11. It is seen that the CDF by the SROM method with size of 9 can recover the general shape of the reference CDF. At the size of 25 , the difference between the SROM-based and reference CDFs becomes very small. On the other hand, the CDF approximated by the SC method is very close to the reference $\mathrm{CDF}$ by using 9
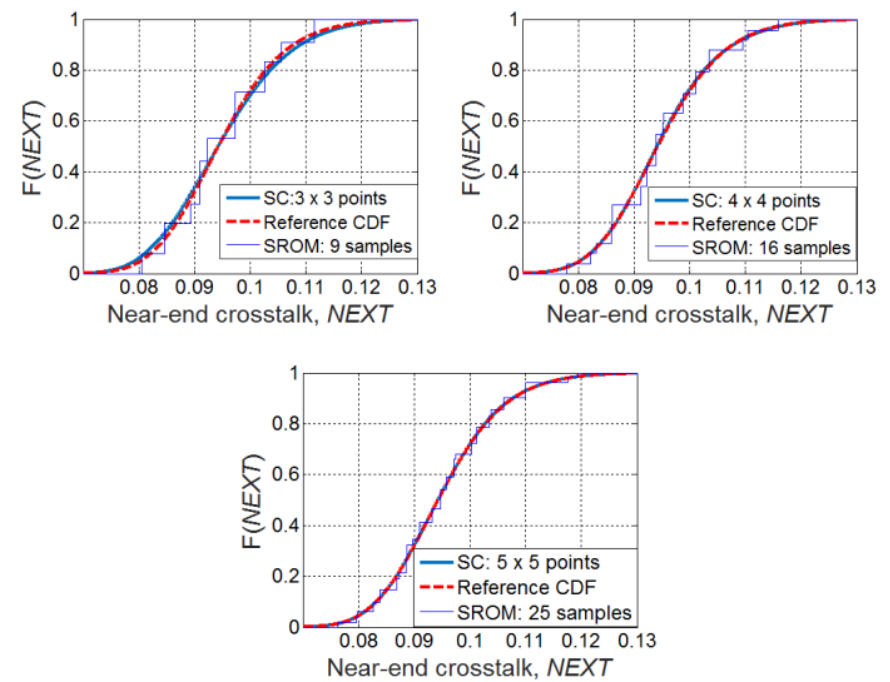

Fig. 11. The reference CDF of NEXT, the CDF approximated by the SC method (using Cubic Hermite interpolating function) with 9,16 and 25 collocation points, and the CDF approximated by the SROMs $\widetilde{N E X T}$ with sizes of 9,16 and 25 .

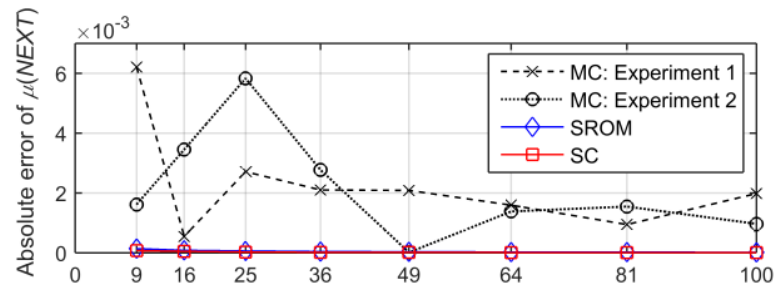

Sample Size of near-end crosstalk, NEXT using SROM, SC and MC

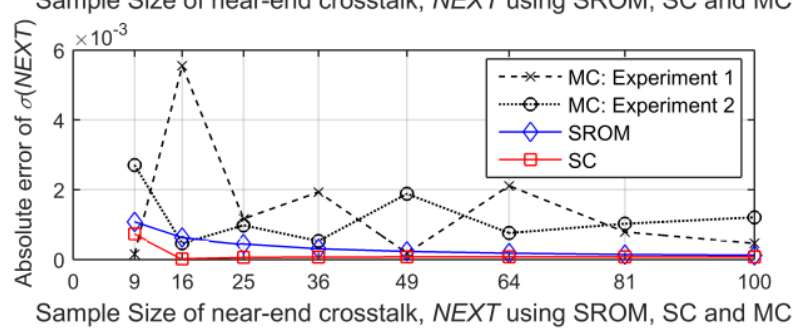

Fig. 12. Convergence rates of the SROM, SC and MC methods under 100 samples, when the random variables are $H$ and $d$.

collocation points. When increasing the number of collocation points to 16, the difference between the SC-based and reference $\mathrm{CDF}$ s becomes indistinguishable.

In Fig. 12, the convergence rates of the SROM, SC and MC methods are compared at the sample sizes (i.e., the number of collocation points for the SC method) of 9 ( $3 \times 3$ for SC), 16 $(4 \times 4), 25(5 \times 5), 36(6 \times 6), 49(7 \times 7), 64(8 \times 8), 81(9 \times 9)$ and $100(10 \times 10)$. It is clear that both the SROM and SC methods steadily converge to the reference result when increasing the sample size, but the convergence rates are different. Specifically, the SROM and SC methods have almost the same performance to predict accurate mean value using small sample sizes, but the convergence rate to the reference standard deviation by the SC method is faster than that by the SROM method. Despite this, the standard deviation by the SROM method is still accurate to a certain extent. For example, at the sample size of 16, the SROM-based standard deviation is within the error of $7 \%$.

Unlike the SC and SROM methods, for the MC method, increasing the sample size may not guarantee the increase in the accuracy of the result. As seen in Fig. 12, the MC method only produces accurate results by incident using small sample sizes, as the approximated statistical results in two MC experiments experience random variations and fail to converge under the sample size of 100 . Therefore, it is clear that for small sample sizes, the MC method only produces different and inaccurate results, whereas the SC and SROM methods are accurate, stable and fast converging.

Fig. 13 shows the variation range of NEXT obtained using the SROM method with sample size of 25 . It can be seen that nearly all the 10,000 MC simulations, except for a small number of extreme cases, are well enclosed by the upper and lower boundaries.

\section{Four Uncertainty Sources}

In this example, the efficacy of the SROM method to recover the statistics of FEXT in the presence of four random variables is demonstrated and compared with that of the SC 


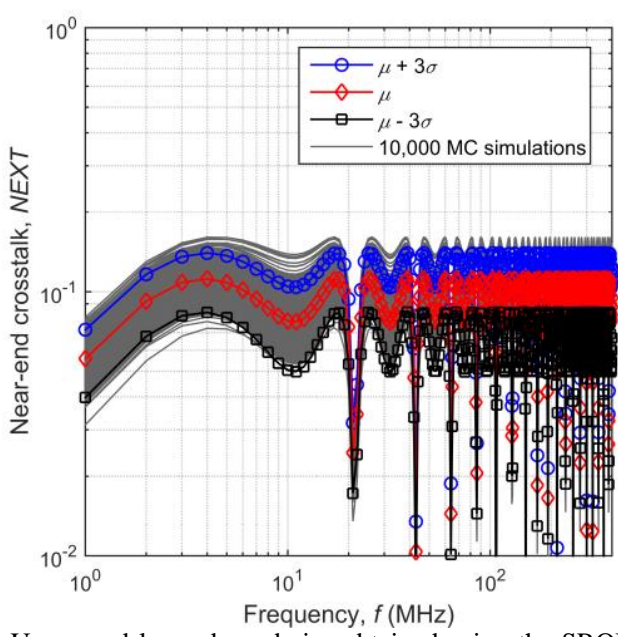

Fig. 13. Upper and lower boundaries obtained using the SROM method to bound the variation of NEXT. At each frequency, only 25 samples of the SROM-based $\widetilde{N E X T}$ are needed. The uncertain variables are $H$ and $d$.

method based on tensor product sampling. The four uncertain variables are selected as the wire height $H$, distance $d$, termination load $T$ and wire radius $r$, following the Gaussian distribution with mean values $E(H)=10 \mathrm{~mm}, E(d)=6 \mathrm{~mm}$, $E(T)=50 \Omega$ and $E(r)=1.024 \mathrm{~mm}$, and standard deviations $\sigma(H)=1 \mathrm{~mm}, \sigma(d)=0.6 \mathrm{~mm}, \sigma(T)=5 \Omega$ and $\sigma(r)=0.1024$ $\mathrm{mm}$. The frequency $f$ and wire length $L$ are assumed to take deterministic values in Table I. In this example, the nominal values of random variables can be different by orders of magnitude. Therefore, this example can demonstrate the potential applicability of the SROM method for stochastic problems where input variables represent different physical quantities.

Let $\boldsymbol{X}$ be a 4-dimensional variable containing all the uncertain variables, i.e., $\boldsymbol{X}=[H, d, T, r]$. In this case, the optimal SROM $\widetilde{\boldsymbol{X}}$ of $\boldsymbol{X}$ cannot be visualized as in the 2dimensional example, but the concept and the construction of the optimal $\widetilde{\boldsymbol{X}}$ follow the same principle. Fig. 14 shows the predicted CDFs of FEXT using the SROM method with the sample size of 50, 81 and 256. It is clear that at the size of 50, the CDF given by the SROM method recovers the general shape of the reference CDF. Then, the difference between the SROM-based and reference CDFs is further reduced at size of 81 , and becomes indistinguishable at size of 256. In order to use the cubic Hermite interpolating function for the SC method, at least 3 collocation points are needed in each random dimension. Therefore, the illustrated SC method based on tensor product sampling needs a minimum of $3^{4}=81$ collocation points in total. If 4 collocation points are selected in each random dimension, the total number of collocation points will be $4^{4}=256$. For the SROM method, choosing the sample size is flexible. As shown in Fig. 14, the CDF predicted by the SC method using 81 samples is almost the same as the reference CDF. Therefore, the SC method may be a better approach to recover the CDF of the system output.

Fig. 15 shows the convergence rates of the SROM method and the SC method using both the linear interpolating function
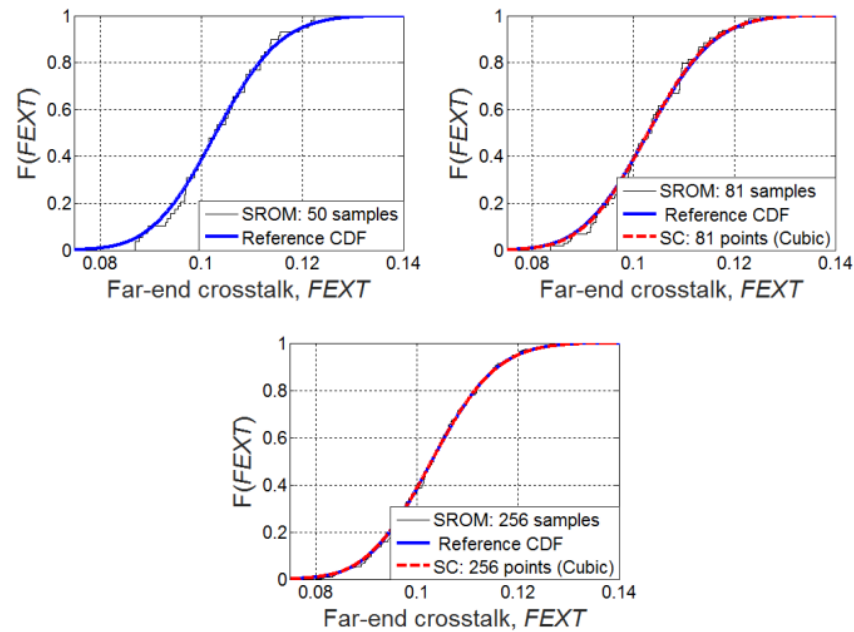

Fig. 14. Comparison of the reference CDF of FEXT, the CDF approximated by the SROMs $\overline{\text { FEXT }}$ with sizes of 50, 81 and 256, and the CDF approximated by the SC method (using Cubic Hermite interpolating function) with 81 and 256 collocation points.

[34] and the cubic Hermite interpolating function. For the SC implementation using linear interpolation and tensor product sampling, the minimum required number of collocation points is $2^{4}=16$, as each random dimension needs at least 2 collocation points. As shown in Fig. 15, the result of the SC method is sensitive to the choice of the interpolating function, as the mean value given by the cubic interpolation is more accurate than that by the linear interpolation. It is also seen in Fig. 15 that both the SROM method and the SC method using the cubic interpolation and tensor product sampling can produce very accurate mean values. In Fig. 15, a steady convergence is observed for the standard deviation by the SROM method, which means a better accuracy is guaranteed by increasing the sample size. We note that the convergence rate of the SROM method to the reference standard deviation is slower than that of the SC method. However, the SROMbased result is still accurate to a certain degree. For example, the standard deviation by the SROM method at sample size of
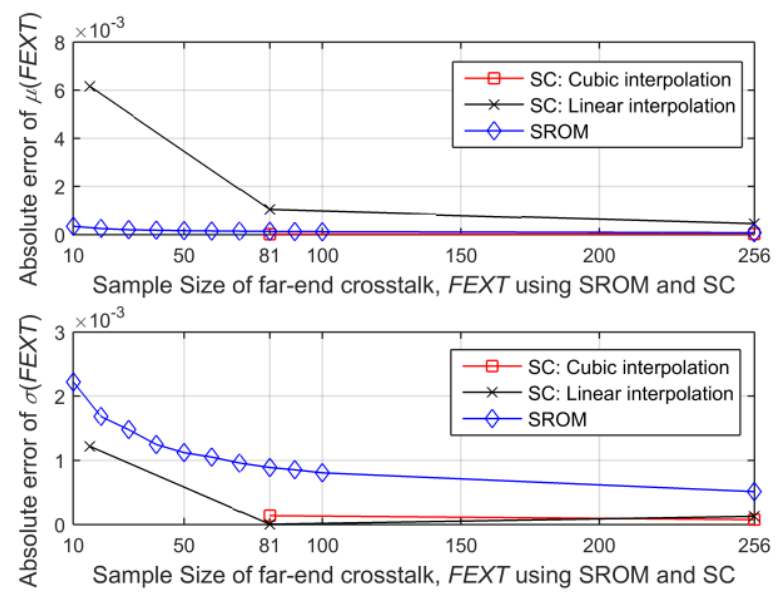

Fig. 15. Convergence rates of the SROM method and the SC method using cubic and linear interpolating functions, when the random variables are $H, d, r$ and $T$. 


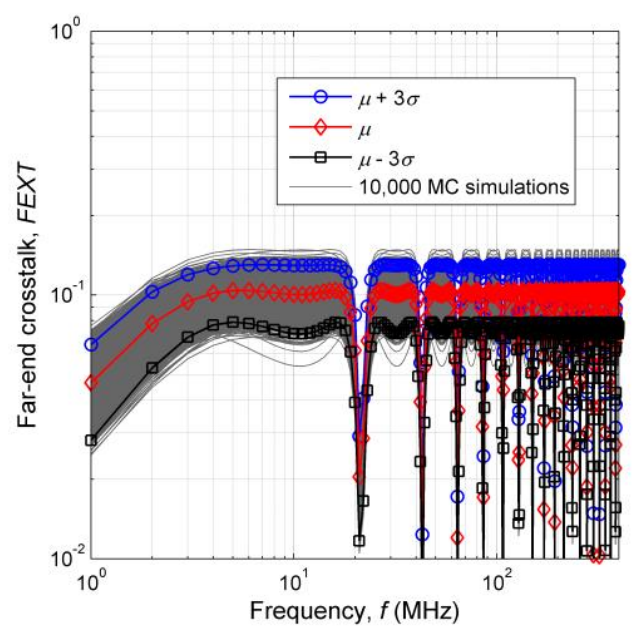

Fig. 16. Upper and lower boundaries obtained with the SROM method to bound the variation of FEXT. At each frequency, only 50 samples of the SROM-based $\widetilde{F E X T}$ are used. The uncertain variables are $H, d, r$ and $T$.

TABLE II EFFICIENCY OF THE SROM AND SC METHODS

\begin{tabular}{|c|c|c|c|c|}
\hline \multicolumn{2}{|c|}{ EXAMPLE } & $\boldsymbol{X}=[H]$ & $\boldsymbol{X}=[H, d]$ & $\boldsymbol{X}=[H, d, r, T]$ \\
\hline \multirow{2}{*}{ SROM } & Time (s) & 0.25 & 0.56 & 4.72 \\
\cline { 2 - 5 } & Samples & 10 & 25 & 81 \\
\hline \multirow{2}{*}{ SC } & Time (s) & 11.03 & 16.57 & 18.48 \\
\cline { 2 - 5 } & Samples & 10 & 25 & 81 \\
\hline \multirow{2}{*}{ MC } & Time (s) & 125.51 & 124.95 & 126.37 \\
\cline { 2 - 5 } & Samples & 10,000 & 10,000 & 10,000 \\
\hline
\end{tabular}

50 is within the error of $10 \%$.

In Fig. 16, the variation range of FEXT is obtained using the SROM method with a sample size of 50. It can be seen that the SROM method can provide accurate upper and lower boundaries to enclose most of the 10,000 MC simulations, except for a small number of extreme occurrences. It is clear that in the case of four uncertainty sources, only a small computational cost is needed to predict the variation range of crosstalk using the SROM method.

From the examples of this study, it is clear that the SC method can produce very accurate statistics of crosstalk using the appropriate interpolating function. On the other hand, the SROM method can provide the mean value of crosstalk as accurate as that of the SC method, but is less accurate than the SC method to predict standard deviation in some cases. Also, choosing the sample size for the SROM method is flexible. The overhead of implementing the SROM and SC methods is also different. Specifically, after the samples of the SROMbased output are obtained, it is straightforward for the SROM method to calculate statistics. For the SC method, having known the output samples at collocation points, the analytical approximation of the output needs to be derived before estimating output statistics. With a CPU of $3.4 \mathrm{GHz}$ and RAM of $8 \mathrm{~GB}$, the computation time of the SROM, SC and MC methods for each example is given in Table II to demonstrate the efficiency of the SROM and SC methods.

We note that it is possible to obtain more accurate results by using other interpolating functions for the SC method. Also, the illustrated SC implementation could be more efficient using sparse grid sampling computed via the Smolyak algorithm. However, such an exhaustive comparison is beyond the scope of this study. The relative goodness of one method over another only holds true in the examples of this study.

There are also some remaining questions about the SROM method itself. Specifically, although a randomness dimensionality of four is tackled using the SROM method in this paper, the maximum randomness dimensionality that the SROM method can handle is still unclear and needs further investigation. Also, it would be beneficial to develop an apriori evaluation method which provides bounds on the errors of the SROM solution. Such an evaluation can be used to select the minimum SROM sample number to keep the computational cost as small as possible whilst guaranteeing sufficient accuracy.

It is worth noting that the demonstration scenario in this study is chosen as a simple three-conductor transmission line, and therefore lacks practical uncertainty sources in a real random bundle. In order to show the efficacy of the SROM method on predicting crosstalk in a realistic cable bundle, one needs to consider typical uncertainty sources discussed in [32], such as the uncontrolled meandering path of each wire, and the presence of dielectric jackets and lacing cords. This is intended as the future work.

\section{CONCLUSIONS}

This paper has introduced a new non-intrusive stochastic approach known as the SROM method to quantify the uncertainties of cable crosstalk. A simple three-conductor transmission line has been taken as the demonstration scenario. The SROM, SC (based on the tensor product sampling strategy) and MC methods have been applied to obtain the statistics of crosstalk subject to multiple uncertainty sources. With the SROM method, the statistics of the actual crosstalk have been accurately approximated, and the variation range of crosstalk has been successfully obtained.

The results from the three methods have been carefully compared and it has been found that the SROM method is more efficient than the MC method, and offers a good accuracy in estimating statistical information. In addition, the sample size for the SROM method has been shown to be flexible depending on the requirement of the result accuracy. It has also been noted that the SC method has a better performance to predict the standard deviation of crosstalk compared with the SROM method. The overhead of the SROM and SC methods has been shown to be different, as the SROM method only needs numerical calculation to obtain the optimal SROM for random variables, whereas the SC method involves algebraic calculation to derive the approximated expression of the output.

Having demonstrated the non-intrusive, accurate, and efficient features of the SROM method in three-conductor transmission lines, the future work is to investigate the advantage of the SROM method to quantify the crosstalk uncertainty subject to practical uncertainty sources in realistic random bundles. In terms of the SROM method itself, the future work can be dedicated to: (1) investigating the maximum dimensionality of the random variable space that the SROM method is practically able to handle; and (2) 
developing an a-priori evaluation of the errors of the SROM solution to choose minimum SROM sample number which guarantees sufficient accuracy.

\section{ACKNOWLEDGMENT}

The authors are grateful for the support and contributions of other members of the ICE-NITE project consortium, from BAE Systems Limited, HORIBA MIRA Ltd, International TechneGroup Ltd, and the University of Nottingham. Further information can be found on the project website (http://www.liv.ac.uk/icenite). The financial support from Innovate UK is gratefully acknowledged.

\section{REFERENCES}

[1] C. R. Paul and A. E. Feather, "Computation of the transmission line inductance and capacitance matrices from the generalized capacitance matrix," IEEE Transactions on Electromagnetic Compatibility, vol. EMC-18, no. 4, pp. 175-183, Nov. 1976.

[2] C. R. Paul, "Useful matrix chain parameter identities for the analysis of multiconductor transmission lines," IEEE Transactions on Microwave Theory and Techniques, vol. 23, no. 9, pp. 756-760, Sep. 1975.

[3] C. R. Paul, Analysis of Multiconductor Transmission Lines, 2nd ed., New York: Wiley, 2008.

[4] Z. Fei, Y. Huang, and J. Zhou, "Crosstalk variations caused by uncertainties in three-conductor transmission lines," in Proc. LAPC, Loughborough, UK, Nov. 2015, pp. 349-353.

[5] S. Shiran, B. Reiser, and H. Cory, "A probabilistic method for the evaluation of coupling between transmission lines," IEEE Transactions on Electromagnetic Compatibility, vol. 35, no. 3, pp. 387-393, Aug. 1993.

[6] S. Pignari, D. Bellan, and L. D. Rienzo, "Statistical estimates of crosstalk in three-conductor transmission lines," in Proc. IEEE Int. Sym. EMC, Minneapolis, USA, Aug. 2002, pp. 877-882.

[7] D. Bellan, S. A. Pignari, and G. Spadacini, "Characterisation of crosstalk in terms of mean value and standard deviation," IEE Proceedings on Science, Measurement and Technology, vol. 150, no. 6, pp. 289-295, Nov. 2003.

[8] C. P. Robert and G. Casella, Introducing Monte Carlo Methods with R, New York: Springer, 2009.

[9] M. S. Halligan and D. G. Beetner, "Maximum crosstalk estimation in lossless and homogeneous transmission lines," IEEE Transactions on Microwave Theory and Techniques, vol. 62, no. 9, pp. 1953-1961, Sep. 2014.

[10] D. Xiu and G. E. Karniadakis, "The Wiener-Askey polynomial chaos for stochastic differential equations," SIAM Journal on Scientific Computing, vol. 24, no. 2, pp. 619-644, Jul. 2002.

[11] D. Xiu, "Efficient collocational approach for parametric uncertainty analysis," Communications in Computatoinal Physics, vol. 2, no. 2, pp. 293-309, Apr. 2007.

[12] I. S. Stievano, P. Manfredi, and F. G. Canavero , "Stochastic analysis of multiconductor cables and interconnects," IEEE Transactions on Electromagnetic Compatibility, vol. 53, no. 2, pp. 501-507, May 2011.

[13] I. S. Stievano, P. Manfredi, and F. G. Canavero, "Parameters variability effects on multiconductor interconnects via Hermite polynomial chaos," IEEE Transactions on Components, Packaging and Manufacturing, vol. 1, no. 8, pp. 1234-1239, Aug. 2011.

[14] D. V. Ginste, D. D. Zutter, D. Deschrijver, T. Dhaene, P. Manfredi, and F. Canavero, "Stochastic modeling-based variability analysis of on-chip interconnects," IEEE Transactions on Components, Packaging and Manufacturing Technology, vol. 3, no. 7, pp. 1244-1251, Jul. 2012.

[15] A. Papoulis, Probability, Random Variables and Stochastic Processes, 3rd ed., New York: McGraw-Hill, 1991.

[16] F. Diouf and F. Canavero, "Crosstalk statistics via collocation method," in Proc. IEEE Int. Sym. EMC, Austin, USA, Aug. 2009, pp. 92-97.

[17] J. E. Warner, W. Aquino, and M. Grigoriu, "Stochastic reduced order models for inverse problems under uncertainty," Computer Methods in Applied Mechanics and Engineering, vol. 285, pp. 488-514, Mar. 2015.

[18] M. Grigoriu, "Reduced order models for random functions. Application to stochastic problems," Applied Mathematical Modelling, vol. 33, no. 1, pp. 161-175, Jan. 2009.
[19] R. V. Field, M. Grigoriu, and J. M. Emery, "On the efficacy of stochastic collocation, stochastic Galerkin, and stochastic reduced order models for solving stochastic problems," Probabilistic Engineering Mechanics, vol. 41, pp. 60-72, Jul. 2015.

[20] M. Grigoriu, "Effective conductivity by stochastic reduced order models (SROMs)," Computational Materials Science, vol. 50, no. 1, pp. 138146, Nov. 2010.

[21] J. E. Warner, M. Grigoriu, and W. Aquino, "Stochastic reduced order models for random vectors: application to random eigenvalue problems," Probabilistic Engineering Mechanics, vol. 31, pp. 1-11, Jan. 2013

[22] M. Grigoriu, "Solution of linear dynamic systems with uncertain properties by stochastic reduced order models," Probabilistic Engineering Mechanics, vol. 34, pp. 168-176, Oct. 2013.

[23] S. Sarkar, J. E. Warner, W. Aquino, and M. Grigoriu, "Stochastic reduced order models for uncertainty quantification of intergranular corrosion rates," Corrosion Science, vol. 80, pp. 257-268, Mar. 2014.

[24] C. R. Paul, "Estimation of crosstalk in three-conductor transmission lines," IEEE Transactions on Electromagnetic Compatibility, vol. 26, no. 4, pp. 182-192, Nov. 1984.

[25] R. Ghanem and P. Spanos, Stochastic Finite Elements: A Spectral Approach. Berlin, Germany: Springer, 1991.

[26] M. Eldred, "Recent advance in non-intrusive polynomial chaos and stochastic collocation methods for uncertainty analysis and design," in Proc. $50^{\text {th }}$ AIAA/ASME/ASCE/AHS/ASC Struct., Structural Dynam., Mat. Conf., Palm Springs, USA, May 2009.

[27] D. Spina, F. Ferranti, T. Dhaene, L. Kockaert, and G. Antonini, "Polynomial chaos-based macromodeling of multiport systems using an input-output approach", International Journal of Numrical modelling: Electronic Network, Devices and Fields, vol. 28, no. 5, pp. 562-581, Sep. 2015.

[28] J.A.S. Witteveen and H. Bijl, "modeling arbitrary uncertainties using Gram-Schmidt polynomial chaos", in Proc. $44^{\text {th }}$ AIAA Aerosp. Sci. Meeting and Exhibit, Reno, USA, Jan. 2006.

[29] P. Manfredi and F.G. Canavero, "General decoupled method for statistical interconnect simulation via polynomial chaos", in Proc. IEEE $23^{\text {rd }}$ Conf. on EPEPS, Portland, USA, Oct. 2014, pp. 25-28.

[30] Q. Du, V. Faber, and M. Gunzburger, "Centroidal voronoi tessellation: applications and algoirthms," SIAM Review, vol. 41, no. 4, pp. 637-676, Oct. 1999.

[31] B. Ganapathysubramanian and N. Zabaras, "Sparse grid collocation schemes for stochastic natural convection problems," Journal of Computational Physics, vol. 225, no. 1, pp. 652-685, Jul. 2007.

[32] D. Bellan and S. A. pignari, "Efficient estimation of crosstalk statistics in random wire bundles with lacing cords," IEEE Transactions on Electromagnetic Compatibility, vol. EMC-53, no. 1, pp. 209-218, Feb. 2011.

[33] F. N. Fritsch and R. E. Carlson, "Monotone piecewise cubic interpolation," SIAM Journal on Numerical Analysis, vol. 17, no. 2, pp. 238-246.

[34] E. Meijering, "A chronology of interpolation: from ancient astronomy to modern signal and image processing," Proceedings of the IEEE, vol. 90, no. 3, pp.319-342, Mar. 2002.

\section{APPENDIX}

This appendix provides a list of the symbols and acronyms in this paper.

\section{TABLE III}

SUMMARIZATION OF THE SYMBOLS IN THIS PAPER

\begin{tabular}{ll}
\hline \hline Symbols & Meanings \\
\hline SROM & Stochastic reduced order model \\
PCE & Polynomial Chaos expansion \\
SG & Stochastic Galerkin \\
SC & Stochastic collocation \\
MC & Monte Carlo \\
$\boldsymbol{X}$ & $d$-dimensional random input variable \\
$\mathrm{F}(\boldsymbol{X})$ & Cumulative distribution function of $\boldsymbol{X}$ \\
$q$ & Moment order \\
$\widetilde{\boldsymbol{X}}$ & Stochastic reduced order model of $\boldsymbol{X}$
\end{tabular}




\begin{tabular}{ll}
$\tilde{\boldsymbol{x}}^{(k)}, k=1, \ldots, m$ & Samples in $\widetilde{\boldsymbol{X}}$ \\
$p^{(k)}, k=1, \ldots, m$ & Probabilities of $\tilde{\boldsymbol{x}}^{(k)}, k=1, \ldots, m$ \\
$\boldsymbol{Y}$ & Output response/solution \\
$\widetilde{\boldsymbol{Y}}$ & Stochastic reduced order model of $\boldsymbol{Y}$ \\
$M$ & Deterministic solver/mapping \\
$N E X T$ & Near-end crosstalk \\
$F E X T$ & Far-end crosstalk \\
$H$ & Height of the conductor \\
$d$ & Distance between two conductors \\
$r$ & Radius of the conductor \\
$T$ & Termination load of the circuit \\
\hline
\end{tabular}

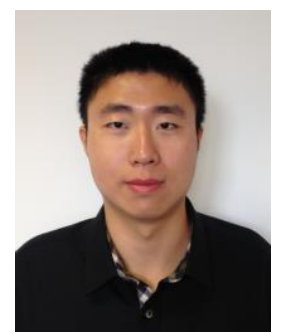

Zhouxiang Fei was born in Xi'an, China, in 1990. $\mathrm{He}$ received his B.Eng. degree in electronics and information engineering from Northwestern Polytechnical University, Xi'an, China, in 2012 and the M.Sc. degree with distinction in wireless communications from the University of Southampton, Southampton, U.K., in 2013. He is currently working toward the Ph.D. degree at the University of Liverpool, Liverpool, U.K.

His research interests include numerical and experimental studies of crosstalk in complex cable bundles, with a particular emphasis on considering parameter variability using efficient statistical approaches.

$\mathrm{He}$ was the recipient of the student scholarship from the IEEE EMC Society to attend the 2016 IEEE International Symposium on EMC, Ottawa, Canada, July 2016. He was also selected as the BEST EMC PAPER FINALIST for the 2016 IEEE International Symposium on EMC.

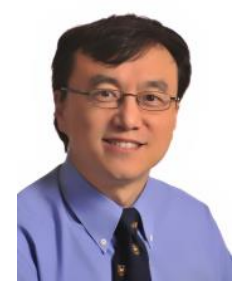

Yi Huang (S'91 - M'96 - SM'06) received BSc in Physics (Wuhan University, China) in 1984, MSc (Eng) in Microwave Engineering (NRIET, Nanjing, China) in 1987, and DPhil in Communications from the University of Oxford, UK in 1994.

$\mathrm{He}$ has been conducting research in the areas of wireless communications, applied electromagnetics, radar and antennas since 1987. His experience includes 3 years spent with NRIET (China) as a Radar Engineer and various periods with the Universities of Birmingham, Oxford, and Essex at the UK as a member of research staff. He worked as a Research Fellow at British Telecom Labs in 1994, and then joined the Department of Electrical Engineering \& Electronics, the University of Liverpool, UK as a Faculty in 1995, where he is now a full Professor in Wireless Engineering, the Head of High Frequency Engineering Group and Deputy Head of Department.

Prof Huang has published over 300 refereed papers in leading international journals and conference proceedings, and is the principal author of Antennas: from Theory to Practice (John Wiley, 2008). He has received many research grants from research councils, government agencies, charity, EU and industry, acted as a consultant to various companies, and served on a number of national and international technical committees and been an Editor, Associate Editor or Guest Editor of four of international journals. He has been a keynote/invited speaker and organiser of many conferences and workshops (e.g. WiCom 2006, 2010, IEEE iWAT 2010, and LAPC2012). He is at present the Editor-in-Chief of Wireless Engineering and Technology, UK National Rep of European Association of Antenna and Propagation, a Senior Member of IEEE, and a Fellow of IET.

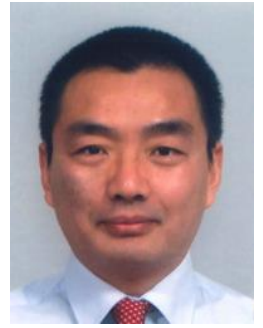

Jiafeng Zhou received a BSc degree in Radio Physics from Nanjing University, Nanjing, China, in 1997, and a Ph.D. degree from the University of Birmingham, Birmingham, U.K., in 2004. His doctoral research concerned high-temperature superconductor microwave filters.

From July 1997, for two and a half years he was with the National Meteorological Satellite Centre of China, Beijing, China. From August 2004 to April 2006, he was with the University of Birmingham, where his research concerned phased arrays for reflector observing systems. Then he moved to the Department of Electronic and Electrical Engineering, University of Bristol, Bristol, U.K until August 2013. His research in Bristol was on the development of highly efficient and linear amplifiers. He is now with the Department of Electrical Engineering and Electronics, University of Liverpool, Liverpool, UK. His past and current research interests include microwave power amplifiers, filters, electromagnetic compatibility, energy harvesting and wireless power transfer.

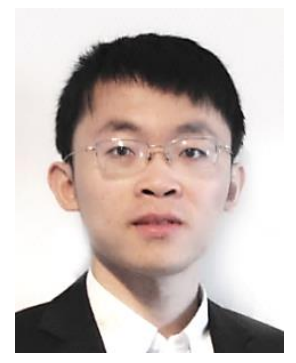

Qian Xu received the B.Eng. and M.Eng. degrees from the Department of Electronics and Information, Northwestern Polytechnical University, Xi'an, China, in 2007 and 2010, and received the $\mathrm{PhD}$ degree in electrical engineering from the University of Liverpool, U.K, in 2016. $\mathrm{He}$ worked as a RF engineer in Nanjing, China in 2011 and an Application Engineer in CST, Shanghai, China in 2012. His research interests include statistical electromagnetics, computational electromagnetics, reverberation chamber and anechoic chamber. 